\title{
- Proteção de dados pessoais e Covid-19: entre a inteligência epidemiológica no controle da pandemia e a vigilância digital
}

\author{
Personal data protection and Covid-19: between epidemiological \\ intelligence in pandemic control and digital surveillance
}

\author{
Carlos Francisco Bitencourt Jorge $\mathrm{a}^{\mathrm{a}, *}$ (1) \\ Bruno Bastos de Oliveiraa (D) \\ João Guilherme de Camargo Ferraz Machadob ${ }^{b}$ \\ Marcelo Souto de Lima ${ }^{\text {(D) }}$ \\ Maria Alice Campagnoli Otrec ${ }^{\text {(D) }}$
}

RESUMO: Trata-se de pesquisa bibliográfica e documental, no contexto da sociedade da informação, que discute a utilização de dados de georreferenciamento para o combate à pandemia da Covid-19. Os problemas de pesquisa gravitam em torno da possibilidade de utilização estatal dos dados privados, sem consentimento prévio, e do equilíbrio entre privacidade e políticas de combate à pandemia. Busca-se refletir sobre a insegurança jurídica que a coleta e armazenamento destes dados oferece ao sistema, concluindo que a Lei Geral de Proteção de Dados (LGPD) pode ser eficiente em relação à proteção de dados pessoais.

Palavras-chave: Proteção de Dados; Inteligência Epidemiológica; Vigilância Digital; Covid-19; São Paulo.

ABSTRACT: This is bibliographic and documentary research, in the context of the information society, which discusses the use of georeferencing data to combat the Covid-19 pandemic. Research problems gravitate towards the possibility of state use of private data, without prior consent, and the balance between privacy and policies to combat the pandemic. It seeks to reflect on the legal uncertainty that the collection and storage of this data offers to the system, concluding that the General Data Protection Law can be efficient in relation to the protection of personal data.

Keywords: Data Protection; Epidemiological Intelligence; Digital Surveillance; Covid-19; São Paulo.

\footnotetext{
a Universidade de Marília, Marília, SP, Brasil.

b Programa de Pós-graduação em Agronegócio e Desenvolvimento, Faculdade de Ciências e Engenharia, Campus Tupã, Universidade Estadual Paulista "Júlio de Mesquita Filho", SP, Brasil.

c Faculdade de Ciências Humanas e Sociais Aplicadas, Universidade de Marília, Marília, SP, Brasil.

*Correspondência para/Correspondence to: Carlos Francisco Bitencourt Jorge. E-mail: bitencourt@gmail.com.

Recebido em/Received: 12/06/2020; Aprovado em/Approved:30/11/2020.
}

Artigo publicado em acesso aberto sob licença $\underline{\mathrm{CC}}$ BY 4.0 Internacional (C)(i) 


\section{INTRODUÇÃO}

Segundo Gaglioni (2020), o uso de celulares no Brasil visando ao combate à pandemia da Covid-19 se assemelha ao uso que tem sido adotado na Europa. Entretanto, na China e na Coreia do Sul, esse uso foi bastante intensificado a partir do uso de aplicativos criados pelos governos para monitorar os passos dos cidadãos de forma individual. Por isso, de modo geral, suscitou a preocupação com a privacidade digital.

Belli (2020) ressalta que a experiência chinesa durante a pandemia oferece relevantes ensinamentos, sobretudo no que se refere à gestão de duas dimensões da informação, que incluem desde a necessidade de comunicação dos riscos e condutas necessárias para conter a doença de maneira clara, efetiva e honesta, até a coleta de informações de qualidade, por serem fundamentais para que medidas mais pertinentes, efetivas e eficientes sejam adotadas.

Trata-se, porém, de rastros de dados deixados pelo caminho, que resultam em grandes bases de dados no ambiente web, e que despertam grande interesse do emprego dessas novas fontes de dados e tecnologias na formulação de políticas públicas (FOLLADOR; RICARD, 2019).

É preciso cuidado para que essa vigilância de emergência pandêmica não se transforme em pretexto para o monitoramento abusivo da população, o que depende do processamento seguro e transparente dos dados necessários para alimentar tal tecnologia, respeitando os direitos individuais e as liberdades coletivas (BELLI, 2020).

As grandes bases de dados inseridas no ambiente web são apontadas como possíveis riscos em relação à proteção de dados pessoais, que por sua vez, estão em grande parte em poder do setor privado (como operadores de telefonia e bancos), impondo desafios éticos e comerciais legítimos para a obtenção de acesso às base de dados coletadas por tais empresas, já que "até o momento, não existem sistemas nem padrões desenvolvidos para que isso seja feito de maneira escalável e de forma ética e segura" (FOLLADOR; RICARD, 2019, p.28).

Melati e Janissek-Muniz (2020, p.4) utilizam o termo 'governo inteligente', para designar "um governo capaz de sentir e reagir ao ambiente, por meio da aquisição de dados a transformar em informações e conhecimentos para o aprimoramento da tomada de decisão no setor público". Os autores destacam que diversos estudos sobre inteligência e governo têm como foco central a segurança nacional, principalmente com foco na necessidade de proteção e desenvolvimento das sociedades e, mais recentemente, com foco nas atividades de segurança pública e de controle de ordem interna.

O monitoramento decorrente dos dados de celulares pode, ao mesmo tempo em que ajuda na prática do isolamento social, permitir a instalação de um 'estado de vigilância', se não tomados alguns cuidados importantes na implantação e condução dessa política pública. O relatório "Privacidade e Pandemia", do Data Privacy Brasil, lançado em 15 de abril de 2020, aponta questões importantes no que se refere ao monitoramento de dados de deslocamento no Brasil, como a ausência de um ciclo de vida e descarte dos dados e de medidas que resguardem a identificação das pessoas, a indefinição de salvaguardas institucionais e lacunas na transparência (BIONI et al., 2020).

É nesse contexto que se mostra fundamental analisar os aspectos jurídicos controvertidos decorrentes da captura e processamento de dados privados, especialmente pela vigência da Lei Geral de Proteção de Dados no Brasil ter se iniciado apenas em setembro de 2020, motivo de grande insegurança jurídica para o sistema, 
que no ápice da pandemia e de expansão da coleta de dados, não estava devidamente regulado.

Com este intuito, a pesquisa se vale de uma abordagem qualitativa, a partir de pesquisa bibliográfica e documental, refletindo na seção 3 sobre o papel central dos dados na Sociedade da Informação e, mais ainda, no acompanhamento e controle da pandemia por Covid-19, por meio da inteligência epidemiológica; e, na seção 4, sobre os aspectos jurídicos que balizam a discussão. A seção 5 aponta algumas considerações sobre este estudo.

\section{PROCEDIMENTOS METODOLÓGICOS}

O presente artigo foi elaborado como base em levantamentos bibliográficos de diferentes temas que, ao se relacionarem, propiciam uma pesquisa com áreas que raramente se relacionam. Marconi e Lakatos (2006, p. 71) mencionam que "[...] a pesquisa bibliográfica não é mera repetição do que foi dito ou escrito sobre certo assunto, mas propicia o exame de um tema sob novo enfoque ou abordagem, chegando a conclusões inovadoras".

Sustentamos que a pesquisa bibliográfica é uma estratégia de pesquisa necessária quando se abordam novos temas, afinal, as relações iniciais podem proporcionar suporte a pesquisas aplicadas. De acordo com Gil (2008, p. 50), a sustentação para as relações entre os temas "é desenvolvida a partir de material já elaborado, constituído de livros e artigos científicos".

Conjuntamente à pesquisa bibliográfica, foram utilizados instrumentos jurídicos que buscaram refletir acerca da revisão teórica, uma vez que no atual cenário pandêmico, as fronteiras entre as leis impostas pela federação, estados e municípios sobre aspectos da sociedade da informação e a sua vigilância, bem como a proteção de seus dados, são questões frágeis e divergentes e carecem de reflexões.

Destaca-se que as reflexões devem ser realizadas acerca dos temas de maneira isolada, como também, na relação e intersecção entre estes temas e os seus resultados. Além disso, o trabalho possui como característica a interdisciplinaridade, pois relaciona diferentes áreas e conceitos (apresentados nas seções seguintes) para construir uma reflexão mais consistente na temática apresentada e que ganhou destaque no momento da pandemia.

\section{SOCIEDADE INFORMACIONAL E MONITORAMENTO ESTATAL}

A sociedade atravessou, ao longo dos séculos, diversos movimentos que foram responsáveis por alicerçar suas atividades. Antes da revolução informacional, estas mudanças eram lentas e firmes, formadas por uma série de situações estáveis que em determinados momentos eram interrompidas por eventos importantes que remodelam os fatos e ajudam a estabelecer a era estável e leve (CASTELLS, 1998; MATTELART, 2002).

A revolução informacional contrapõe todo esse movimento estável e suave, sendo esta apoiada pelo advento das Tecnologias da Informações e Comunicações (TICs). $\mathrm{Na}$ 'Sociedade da Informação', cada organização passa a ser uma organização de informação, e cada organismo é um organismo de informação, tornando a informação um recurso necessário para organizar o que a sociedade desenvolve e constrói (BELL, 1979 apud MATTELART, 2002). 
Mattelart (2002), destaca que os avanços tecnológicos na expressão e transmissão de informações aceleram a desigualdade social, a partir da construção de "monopólios informacionais", utilizados como instrumentos para se os detentores das novas tecnologias tornarem-se dominantes em vários aspectos sociais, inclusive na política.

A sociedade digital passa por um processo de assimilação que gera novas formas de interação social e modifica práticas antes consolidadas, alterando os modos de vida social (MACHADO; DIAS; FERRER, 2018). Drucker (1969, 2000) cunhou o termo 'economia do conhecimento' e 'sociedade do conhecimento', trazendo assim o conhecimento como importante recurso social e econômico da sociedade.

Sendo a informação o recurso central da sociedade da informação, a Internet passa a centralizar este processo provendo dados de diversas naturezas, possibilitando caracterizar a sociedade e seus comportamentos, que podem ser encontrados em diferentes locais como mídias online, sites governamentais, fóruns, blogs, redes sociais e, indiretamente, por meio do geoprocessamento, capturando dados e monitorando ações realizadas nos mundos virtual e real.

Estes dados, atualizados em tempo real, constroem inúmeros cenários em relação ao comportamento da sociedade e, atualmente, mostram-se como fundamentais no combate à pandemia do Covid-19 (SARS-CoV-2), mapeando a transmissão da doença e auxiliando áreas geográficas com limitada infraestrutura de saúde pública.

A Inteligência Epidemiológica (IE), que visa a proteção do estado de bem-estar de populações ameaçadas por evento ou comportamento indesejados, como no caso das epidemias, utiliza-se de ferramentas que possibilitem a coleta, validação e análise de informações para a construção de cenários e conhecimentos sobre a sociedade, tornando possível o desenvolvimento de ações e estratégias de saúde pública (RODRIGUES-JÚNIOR, 2012; WILBURN et al, 2019; CHEN; CHUGHTAI; MACINTYRE, 2020).

O cenário mundial de enfrentamento à Covid-19, iniciada em dezembro de 2019, na China, é propício para o uso dessas ferramentas (MCKIBBIN; FERNANDO, 2020). No Brasil, o primeiro caso da Covid-19 surgiu em 26 de fevereiro de 2020 e, de acordo com a Organização Mundial da Saúde (OMS), rapidamente o estado de São Paulo transformou-se no epicentro da pandemia no país, local onde inicialmente foi utilizada a tecnologia de geoprocessamento na tentativa do controle da pandemia por meio do isolamento social. Dados do Ministério da Saúde indicam 5.323.630 casos confirmados no país e 155.900 óbitos confirmados em 23 de outubro de 2020.

O alastramento do vírus por todo o planeta levou a OMS a indicar o isolamento social como um dos principais pilares estratégicos para contenção da pandemia. Dessa maneira, uma parte significativa da sociedade mundial, seguindo recomendações de seus governantes, permanece em suas casas de maneira voluntária ou compulsória para não contraírem o vírus e/ou não transmiti-lo a outras pessoas.

Ao seguir as diretrizes da Organização Mundial da Saúde (OMS), o Governo do Estado de São Paulo decretou estado de calamidade (Decreto $N^{\circ} 64.881^{1}$ ) no dia 22 de março de 2020, 26 dias após o surgimento primeiro caso no estado. Essa decisão impactou todos os 645 municípios, ao colocar em quarentena (iniciada no dia 24 de março) toda a população paulista, com a exceção dos trabalhadores essenciais.

\footnotetext{
1 DECRETO No 64.881, DE 22 DE MARÇO DE 2020. Disponível em: https://www.saopaulo.sp.gov.br/wpcontent/uploads/2020/03/decreto-quarentena.pdf.
} 
No início de maio de 2020, o governo do Estado de São Paulo instituiu o Sistema de Monitoramento Inteligente (SIMI), por meio do Decreto $\mathrm{N}^{\circ} 64.963^{2}$, resultado de um acordo com as quatro principais operadoras de telefonia móvel no estado, objetivando utilizar dados georreferenciais para monitorar o percentual de pessoas em isolamento social e identificar áreas com aglomerações, possibilitando a realização de ações de advertência e orientação (CORRÊA; LUZ, 2020). O decreto destaca que o SIMI deve apoiar a formulação e a avaliação das ações do Estado de São Paulo no enfrentamento da pandemia sem utilização de dados pessoais, limitando-se apenas aos dados anonimizados.

Segundo a Secretaria Especial de Comunicação do Estado de São Paulo, os dados de telefonia móvel são analisados pela central de inteligência, apontando tendências de deslocamento e a eficácia das medidas de isolamento social, permitindo a intensificação de campanhas de conscientização com apoio das prefeituras, por ser possível identificar em quais regiões a adesão à quarentena é maior ou menor (COMUNICAÇÃO, 2020).

O processo de isolamento social foi incentivado pela maioria dos estados brasileiros, que realizam o monitoramento por meio do geoprocessamento, a partir de dados fornecidos pelas operadoras de telefonia celular. Com isso, surgem importantes questionamentos referentes à privacidade dos dados da sociedade digital: 1) $O$ estado pode utilizar dos dados sem autorização prévia dos sujeitos que compõem a sociedade? e 2) Até que ponto a privacidade da sociedade está acima dos aspectos de saúde em situações de calamidade, como no caso da pandemia de Covid-19?

\section{ASPECTOS JURÍDICOS CONTROVERTIDOS ACERCA DA VIGILÂNCIA DIGITAL}

A sociedade informacional surge a partir de processos de inovação que formam complexas relações e necessitam do acompanhamento e amadurecimento do processo de formação regulatória, sem desconsiderar diferenças culturais, sociais e econômicas, inerentes a este novo modelo social.

Neste modelo, a tecnologia e a sociedade se completam e, embora uma não determine a outra, interfere nos seus rumos. Entretanto, para Castells (1998), uma sociedade não pode ser retratada ou classificada sem suas ferramentas tecnológicas.

O debate envolvendo a tecnologia empregada no enfrentamento à Covid-19 esbarra na coleta e utilização de dados pessoais e nas possíveis violações a direitos fundamentais. A indispensabilidade de proteção de dados pessoais, apresenta-se como necessidade própria do processo de digitalização social, onde informações, caracterizadas por dados, passam a ter alta relevância política, econômica e social (GEDIEL; CORRÊA; 2008). Destarte, os impactos produzidos pelo combate à pandemia instigaram ainda mais o debate acerca da proteção de dados.

A coleta de dados pessoais para a IE aquece a discussão acerca da proteção de informações digitais e a necessidade de marcos regulatórios claros que definam os limites para sua utilização, sendo necessário entender as transmutações ocorridas nesta sociedade com a consequente e necessária intervenção do direito para prescrever estas novas relações (CALHEIROS; TAKADA; 2015).

\footnotetext{
2 DECRETO No 64.963, DE 05 DE MAIO DE 2020. Disponível em: https:/www.saopaulo.sp.gov.br/wp-
} content/uploads/2020/05/Decreto-64963-de-05-de-maio-de-2020-SIMI.pdf. 
Na sociedade da informação, a proteção de dados pessoais necessita de norma que regulamente tal relação, já que para o manuseio desta informação se fará necessária a obediência de regras específicas, bem como a anuência do titular destes dados, que permitirá o que pode e o que não pode ser realizado com tais informações.

A discussão acerca da coleta de dados pessoais trata diretamente dos direitos fundamentais, mas é inegável que o correto tratamento destes dados pessoais fornece importantes informações estratégicas no combate à pandemia, por meio da criação e aprimoramento de políticas de saúde pública.

A fim de evitar excessos no manuseio desses dados pessoais, tem-se como instrumento delimitador à dignidade da pessoa humana que, enquanto princípio fundamental, se apresenta como um dos pilares do Estado Democrático de Direito, estabelecida pelo legislador como essência qualitativa de todo e qualquer fracionamento jurídico de direito privado (MULHOLLAND, 2018).

No Brasil, a regulação de dados pessoais está prevista na Lei 13.709/2018, mais conhecida como Lei Geral de Proteção de Dados (LGDP), cuja vigência se deu a partir de 18 de setembro de 2020. Já as penalidades previstas pela Lei só poderão ser aplicadas a partir de agosto de 2021, sendo esta alteração resultado direto da pandemia provocada pelo Covid-19, que instaurou grande celeuma acerca da vigência do referido instrumento legal.

Mesmo com a aplicação de sanções suspensas, a LGPD oferece ao titular dos dados o direito à privacidade e à vida íntima, por meio da manutenção das garantias constitucionais estabelecidas no processo de captação, compartilhamento, tratamento e armazenamento de dados pessoais, com o estabelecimento de instrumentos de governança da segurança das informações (VIEIRA, 2019).

A coleta de dados pessoais e a proteção destes é importante a qualquer tempo. Ocorre que a necessidade de transparência acerca da finalidade e do tratamento destinado a estes dados é de fundamental importância tanto para o momento atual quanto para o pós-pandemia.

A IE vem utilizando o geoprocessamento para avaliar a mobilidade populacional, eventuais pontos de aglomeração e, com isso, a adesão da sociedade ao isolamento social, conforme release publicado pelo Sindicato Nacional da Empresas de Telefonia e de Serviço Móvel Celular e Pessoal (SindiTelebrasil).

Em movimento semelhante, o Governo Federal publicou em 17 de abril a Medida Provisória 954/2020, que dispõe sobre o compartilhamento de dados por empresas de telecomunicações com o Instituto Brasileiro de Geografia e Estatística (IBGE), visando a realização de entrevistas não presenciais no desenvolvimento de pesquisas domiciliares. Todavia, a eficácia dessa Medida Provisória (MP) foi suspensa pelo Supremo Tribunal Federal, que entendeu existir risco de violação à princípio privacidade.

Tais medidas, direta ou indiretamente relacionadas ao combate da pandemia, necessitam de regulação, principalmente do processo de coleta desses dados pessoais. Vieira (2019) destaca que a LGPD ao longo do processo de utilização da informação, veda que o titular de tais dados seja identificado ou identificável.

Percebe-se que a falta de um instrumento regulatório ao longo dos primeiros meses da pandemia prejudicou a proteção de dados, situação que foi acentuada pelo fato de ainda não estar constituído o órgão regulador e fiscalizador da proteção de dados pessoais, a Autoridade Nacional de Proteção de Dados (ANPD). 
Enquanto instrumento regulatório, a LGPD traz importantes ferramentas no tocante à proteção de dados pessoais, destacando-se a finalidade (artigo $6^{\circ}, 1$ ); necessidade (artigo $6^{\circ}$, III); transparência (artigo $6^{\circ}, \mathrm{VI}$ ) e prestação de contas (artigo $6^{\circ}, \mathrm{X}$ ). Merece destaque também a necessidade do consentimento do titular dos dados, conforme 0 artigo $7^{\circ}$, I, para que haja o tratamento dos mesmos.

Em análise comparativa, a General Data Protection Regulation (GDPR), a Lei de Proteção de Dados Europeia que inspirou a LGPD em muitos aspectos, garante aos cidadãos da Comunidade Europeia proteção, responsabilizando o operador e controlador, caso os dados coletados para fins de geoprocessamento e combate à pandemia tenham destino diferente daquele informado ao usuário.

Se estivesse vigorando desde o início da pandemia, a LGPD seria instrumento ideal para coibir abusos, não apenas por estabelecer os meios pelos quais devem ocorrer a coleta de dados, mas por estabelecer infraestrutura indispensável ao desenvolvimento da sociedade informacional, responsabilizando o controlador e operador destes dados, além de permitir completo acesso ao cidadão e, sobretudo, controle dos seus dados (BAIÃO; GONÇALVES; 2014).

\section{REFLEXÕES E CONSIDERAÇÕES PARCIAIS}

O governo de São Paulo faz uso de duas importantes estratégias complementares: a Análise Espacial ( $A E)$ e o Processo de Inteligência. Nesse sentido, destaca-se que a AE, enquanto processo, proporciona a mensuração de propriedades e relacionamentos, considerando a localização espacial do fenômeno analisado em estudo explícito. Para tanto, vem utilizando modelos espaciais analíticos compostos por um conjunto de procedimentos, com o objetivo de descrever um fenômeno por meio de suas relações com o espaço geográfico (MONTEIRO et al, 2004). Enquanto isso, a Inteligência é compreendida como um processo responsável por coletar e analisar os dados, construindo assim informações que ao serem disseminadas tornam-se inteligência (MILLER, 2002).

Cepik (2002) propõe uma dualidade que constitui contraposições capazes de dividir opiniões: em uma abordagem estratégica da sociedade de informação, a inteligência se refere a toda informação coletada, organizada e analisada, visando a tomada de decisões. Por outro lado, em uma abordagem restritiva (considerando o poder coercitivo do Estado), refere-se à inteligência como a coleta de informações sem o consentimento, sem a cooperação ou, ainda, sem o conhecimento por parte cidadão, com uma finalidade investigativa. Segundo Jorge e Araújo (2020), a aplicação dos processos de maneira conjunta na saúde não é algo novo, esse uso conjunto vem sendo realizado desde o século XIX.

Diante dessa situação que tramita entre as esferas da saúde pública e do direito, questiona-se, no atual estado de pandemia e com inúmeros recursos tecnológicos, se o armazenamento e uso desses dados serão apenas durante a pandemia ou se serão utilizados a posteriori?

Importante destacar que o estudo não cogita que a Administração Pública não tenha acesso aos dados pessoais, especialmente para o período de combate dessa pandemia de proporções grandiosas, contudo deve haver garantias ao titular de que seus dados não serão utilizados senão para auxiliar no combate à Covid-19.

Parece evidente que, em períodos excepcionais como o vivido atualmente, o Poder Legislativo se coloca como importante instrumento de criação de condições favoráveis para que o país possa ter mecanismos adequados de enfrentamento da crise. E é 
justamente por este motivo que se entende que a existência de instrumento regulatório, caracterizado pela LGPD, era de extrema necessidade desde o início da pandemia.

A LGPD é o instrumento regulatório específico no tocante à proteção de dados pessoais, o início de sua vigência, ainda que de forma tardia, traz o conforto vinculado ao consentimento do titular do dado, no tocante à forma e finalidade na utilização da referida informação, uma vez que há a expectativa de privacidade assegurada ao usuário por meio da lei, protegendo o titular dos dados pessoais contra qualquer ingerência externa (ARAUJO; CAVALHEIRO, 2014).

Assim, além do instrumento regulatório atual, faz-se necessário, com urgência, a constituição da competente agência reguladora, para garantir que os dados pessoais coletados em virtude da pandemia não serão tratados ou compartilhados sem o consentimento do cidadão. Segundo Goldschmidt e Reis (2018), a proteção de dados pessoais em uma sociedade informacional decorre do direito à privacidade e a vida íntima do cidadão, sendo fundamental a garantia de tal tutela.

Afinal, existe uma grande quantidade de dados pessoais que até o momento estão sendo coletados sob o argumento de combate à Covid-19. O armazenamento de tais informações em um cenário extremo poderia ensejar um estado de vigilância social? Em momento algum colocou-se em xeque a necessidade dos dados para uso e construção de estratégias de saúde pública, porém, no início da pandemia não seria correto ter-se adiantado a implementação da LGPD ao invés de adiá-la, nem que fosse com enfoque no estado?

A presente pesquisa buscou realizar uma reflexão diante as ações do estado frente ao cenário de pandemia, bem como o impacto dessas ações na sociedade. Recomenda-se futuras pesquisas que realizem o acompanhamento e observem a destinação dos dados que estão sendo coletados e usados pelos estados, em especial o de São Paulo.

\section{REFERÊNCIAS}

ARAUJO, L. E. B.; CAVALHEIRO, L. N. A proteção de dados pessoais na sociedade informacional brasileira: o direito fundamental à privacidade entre a autorregulação das empresas e a regulação protetiva do internauta. Revista do Direito Público, Londrina, v. 9, n. 1, p. 209-226, jan./abr. 2014. Disponível em http://www.uel.br/revistas/uel/index.php/direitopub/article/view/16870. Acesso em: 26 mar. 2020.

BAIÃO, K. C. S.; GONÇALVES, K. C. A garantia da privacidade na sociedade tecnológica: um imperativo à concretização do princípio da dignidade da pessoa humana. Civilistica.com, ano 3, n. 2, p. 1-24, 2014. Disponível em: http://civilistica.com/a-garantia-da-privacidade-na-sociedade-tecnologica-umimperativo-a-concretizacao-do-principio-da-dignidade-da-pessoa-humana/. Acesso em: 30 mar. 2020

BELLI, L. Os big data do coronavírus. El País, 09 mar. 2020. Disponível em https://brasil.elpais.com/opiniao/2020-03-09/os-big-data-do-coronavirus.html. Acesso em: 18 abr. 2020.

BIONI, B.; ZANATTA, R.; MONTEIRO, R.; RIELLI, M. Privacidade e pandemia: recomendações para o uso legítimo de dados no combate à COVID-19. Conciliando o combate à COVID-19 com o uso legítimo de dados pessoais e o respeito aos direitos 
fundamentais. São Paulo: Data Privacy Brasil, 2020. Disponível em https://www.dataprivacybr.org/wp-content/uploads/2020/04/Relatorio-Privacidade-ePandemi-a-Data-Privacy-Brasil-2.pdf. Acesso em: 10 maio 2020.

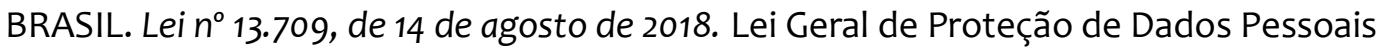
(LGPD). Brasília, DF, Presidência da República. Disponível em: http://www.planalto.gov.br/ccivil_03/_at02015-2018/2018/lei/L13709.htm. Acesso em: 28 mar. 2020.

CALHEIROS, T. C.; TAKADA, T. A. Reflexões sobre a privacidade na sociedade da informação. Inf. Prof., v. 4, n.1, p.120-134, 2015. Disponível em http://www.uel.br/revistas/uel/index.php/infoprof/article/view/22564/17209. Acesso em: 25 mar. 2020.

CASTELLS, M. A sociedade em rede: a era da informação: economia, sociedade e cultura. São Paulo: Ed. Paz e Terra, 1998.

CHEN, X.; CHUGHTAI, A. A.; MACINTYRE, C. R. Application of a risk analysis tool to middle east respiratory syndrome coronavirus (MERS-CoV). Outbreak in Saudi Arabia. Risk Analysis, v. 40, p. 915-925, May 2020. Disponível em https://doi.org/10.1111/risa.13472. Acesso em: 28 de maio de 2020.

CHOO, C. W. A organização do conhecimento: como as organizações usam a informação para criar significado, construir conhecimento e tomar decisões. São Paulo: Senac São Paulo, 2003.

CORRÊA, A. E.; LUZ, P. H. M. A exceção na proteção de dados pessoais durante a Covid-19 - parte 1. ConJur, 22 maio 2020. Disponível em: https://www.conjur.com.br/2020-mai-22/direito-civil-atual-excecao-protecao-dadospessoais-durante-covid-19\#_ftn10. Acesso em: 23 out. 2020.

DRUCKER, P. The age of discontinuity: guidelines to our changing society. New Brunswick: Transaction Books, 1969.

DRUCKER, P. Além da revolução da informação. HSM Management, ano 4, n. 18, jan./fev. 2000. Disponível em http://www.strategia.com.br/Arquivos/Al\%Egm_da_revolu\%E7\%E30_da_informa\%E7\%E 30.pdf. Acesso em: 16 mar. 2020.

MONTEIRO, A. M. V.; CÂMARA, G.; CARVALHO, M. S.; DRUCK, S. Análise espacial de dados geográficos. Brasília: Embrapa, 2004. Disponível em:

http://www.dpi.inpe.br/gilberto/livro/analise/. Acesso em: 05 de abril de 2020.

FOLLADOR, S. R.; RICARD, J. Big data para o bem comum. GVExecutivo, v.18, n. 4, p. 26-29, 2019. Disponível em: http://dx.doi.org/10.1266o/gvexec.v18n4.2019.79976. Acesso em: 13 maio 2020.

GAGLIONI, C. Os cuidados com o uso de dados móveis na pandemia. Nexo, 15 abr. 2020. Disponível em: https://www.nexojornal.com.br/expresso/2020/04/15/Oscuidados-com-o-uso-de-dados-m\%C3\%B3veis-na-pandemia-segundo-esterelat\%C3\%B3rio. Acesso em: 20 abr. 2020.

GIL, A. C. Métodos e técnicas da pesquisa social. 6.ed. São Paulo: Atlas, 2008. 
GEDIEL, J. A. P.; CORRÊA, A. E. Proteção jurídica de dados pessoais: a intimidade sitiada entre o estado e o mercado. Revista da Faculdade de Direito - UFPR, Curitiba, n. 47, p. 141-153, 2008. Disponível em: https://revistas.ufpr.br/direito/article/view/15738/10444. Acesso em: 18 mar. 2020.

GOLDSCHMIDT, R.; REIS, B. F. A proteção dos dados pessoais e sensíveis do empregado no ordenamento jurídico brasileiro frente à sociedade da informação: uma abordagem sob a teoria da eficácia horizontal dos direitos fundamentais. In: WOLKMER, A. C.; VIEIRA, R. S. (Org.). Direitos humanos e sociedade. Santa Cruz do Sul: Essere nel Mondo, 2018. V.1.

JOHNS HOPKINS UNIVERSITY. Coronavirus Resource Center. 2020. Disponível em: https://coronavirus.jhu.edu/map.html. Acesso em: 17 abr. 2020.

JORGE, C. F. B.; ARAUJO, A. S. de. Análise espacial no processo de inteligência competitiva como estratégia para a construção de produtos e serviços de inteligência voltados às organizações. Ciência da Informação, v. 48, n. 1, p. 218-234, jan./abr. 2019. Disponível em: http://revista.ibict.br/ciinf/article/view/4287/4107. Acesso em: 23 maio 2020.

MACHADO, V. R. P.; DIAS, J. A.; FERRER, W. M. H. Biopolítica e novas tecnologias: o discurso do ódio na Internet como mecanismo de controle social. Revista de Informação Legislativa, v. 55, n. 220, p. 29-51, out./dez. 2018. Disponível em: http://www12.senado.leg.br/ril/edicoes/55/220/ril_v55_n220_p29. Acesso em: 03 maio 2020.

MARCONI, M. A.; LAKATOS, E. M. Técnicas de pesquisa: planejamento e execução de pesquisas, amostragens e técnicas de pesquisa, elaboração, análise e interpretação dos dados. São Paulo: Atlas, 2006.

MATTELART, A. História da sociedade da informação. São Paulo: Loyola, 2002.

MCKIBBIN, W. J.; FERNANDO, R. The global macroeconomic impacts of COVID-19: seven scenarios. CAMA Working Paper, n. 19, p. 1-43, mar. 2020. Disponível em: http://dx.doi.org/10.2139/ssrn.3547729. Acesso em: 08 de jun. de 2020.

MELATI, C.; JANISSEK-MUNIZ, R. Governo inteligente: análise de dimensões sob a perspectiva de gestores públicos. Revista de Administração Pública, Rio de Janeiro, v. 54, n. 3, p. 400-415, abr. 2020. Disponível em: http://bibliotecadigital.fgv.br/ojs/index.php/rap/article/view/81259. Acesso em: 23 maio 2020.

MILLER, J. P. O processo de inteligência: como funciona, seus benefícios e sua situação atual. In: MILLER, J. P. O milênio da inteligência competitiva. Porto Alegre: Bookman, 2002. p. 32-52.

MULHOLLAND, C. S. Dados pessoais sensíveis e a tutela de direitos fundamentais: uma análise à luz da lei geral de proteção de dados (lei 13.709/18). Estado de Direito e Tecnologia (Edição Temática), v. 19, n. 3, p. 159-180, 2018. Disponível em: https://www.researchgate.net/publication/330000896_Dados_pessoais_sensiveis_e_ a_tutela_de_direitos_fundamentais_uma_analise_a_luz_da_lei_geral_de_protecao_ de_dados_Lei_1370918. Acesso em: 18 maio 2020. 
OPERADORAS vão disponibilizar dados de mobilidade ao MCTIC para monitorar deslocamento. Sinditelebrasil, 2020. Disponível em https://www.sinditelebrasil.org.br/sala-de-imprensa/releases/3375-operadoras-vaodisponilizar-dados-de-mobilidade-ao-mctic-para-monitorar-deslocamento. Acesso em: 02 abr. 2020.

ORGANIZAÇÃO MUNDIAL DA SAÚDE. Coronavirus disease (COVID-19) Pandemic. Disponível em: https://www.who.int/emergencies/diseases/novel-coronavirus-2019. Acesso em: 10 jun. 2020.

RODRIGUES-JÚNIOR, A. L. A inteligência epidemiológica como modelo de organização em saúde. Ciência \& Saúde Coletiva, v.17, p. 797-805, 2012. Disponível em: https://www.scielosp.org/article/csc/2012.v17n3/797-805/. Acesso em: 13 abr. 2020.

SANTOS, L. A. D. C. Um século de cólera: itinerário do medo. Physis, Rio de Janeiro, v. 4, n. 1, p. 79-110, 1994. Disponível em: http://dx.doi.org/10.1590/S010373311994000100005. Acesso em: 10 mar. 2020.

SÃO PAULO. Secretaria Especial de Comunicação. Covid-19: capital registrou 43\% de índice de isolamento social na segunda-feira (24). 25 ago. 2020. Disponível em: http://www.capital.sp.gov.br/noticias/capital-registrou-43-de-indice-de-isolamentosocial-na-segunda-feira-24. Acesso em: 23 out. 2020.

VALENTIM, M. L. P. Inteligência competitiva em organizações: dado, informação e conhecimento. DataGramaZero, v. 3, n. 4, p. 1-13, 2002. Disponível em: https://www.brapci.inf.br/_repositorio/2010/01/pdf_f589d25523_0007468.pdf. Acesso em: 20 mar. 2020.

VIEIRA, E. L. C. A proteção de dados desde a concepção (by design) e por padrão (by default). In: MALDONADO, V. N. (Coord.). LGPD: Lei Geral de Proteção de Dados pessoais: manual de implementação. São Paulo, Thomson Reuters Brasil, 2019.

WILBURN, J.; O'CONNOR, C.; WALSH, A. L.; MORGAN, D. Identifying potential emerging threats through epidemic intelligence activities: looking for the needle in the haystack?. International Journal of Infectious Diseases, v. 89, p. 146-153, 2019. Disponível em: https://doi.org/10.1016/j.ijid.2019.10.011. Acesso em: 13 jun. 2020 